\title{
Los residuos agroindustriales en la construcción civil en Brasil
}

\footnotetext{
Holmer Savastano Jr. ${ }^{*}$, Samuel Rosario F.**, Carlo A. Flores $^{* * *}$, Reginaldo Araujo****

* Ingeniero civil por la Escuela Politécnica de la Universidad de São Paulo, Brasil. ** Ingeniero químico por la Universidad Nacional Mayor de San Marcos. Profesor en la Escuela de Post-grado de la Universidad Nacional de Ingeniería. Profesor de la Especialidad de Ingeniería Metalúrgica y de Materiales de la Universidad de Lima. ${ }^{* * *}$ Estudiante de Ingeniería de Agrimensura-Pirassununga, São Paulo, Brasil. **** Estudiante de la Especialidad de Ingeniería Metalúrgica y de Materiales de la Universidad
} de Lima.

El objetivo del presente trabajo es analizar la posibilidad de aprovechamiento de residuos y subproductos de la extracción y del procesamiento de fibras vegetales para reciclaje, en materiales para la construcción civil en Brasil. Realizamos visitas técnicas a los principales polos generadores de fibras de las regiones norte, noreste, sureste y sur del Brasil. Sobre la base de criterios preestablecidos fueron seleccionados cinco diferentes tipos de residuo, a partir de las fibras de sisal (Agave sisalana), coco (Cocos nucifera), pulpa de celulosa de eucalipto (Eucalyptus grandis) y banana (Musa canvendishii ). Esos residuos fueron caracterizados y utilizados en el refuerzo de matrices frágiles basándose en cemento de escoria básica granulada de alto horno. Los resultados demostraron la potencialidad de los desechos estudiados, teniendo en cuenta principalmente la gran disponibilidad, bajo valor de mercado y propiedades mecánicas de los compósitos resultantes, significativamente superiores a las de la matriz sin refuerzo. 


\section{Introducción}

El potencial productivo de fibras orgánicas, en el Brasil, es muy elevado, habiendo sido analizados, por V. Agopyan en 1991, cerca de veinte tipos útiles en la construcción civil. Los residuos generados por la agroindustria de la fibra vegetal pueden constituir, por sí mismos, una importante fuente de materia prima para refuerzo de componentes constructivos, dependiendo de las cantidades disponibles y de la dispersión geográfica (John, 1996).

Las fibras vegetales estudiadas en el presente trabajo fueron escogidas porque constituyen una gran fuente de residuos agroindustriales. Además de eso, algunas de ellas (sisal, principalmente) tienen importancia social significativa, tomando en cuenta la generación de empleo que proporcionan en regiones agrícolas muy subdesarrolladas.

Así, por su bajo costo, las fibras vegetales pasaron a ser investigadas como alternativas destinadas a las construcciones de interés social, al ser empleadas cerca de las regiones de origen como desecho de otras aplicaciones comerciales.

En lo que respecta a la durabilidad de los compósitos fibrosos, algunos investigadores proponen el tratamiento de las fibras vegetales por medio de polímeros e hidrorrepelentes (resinas, aceites y asfaltos), como forma de protegerlas de la agresión del medio (Guimarães, 1990). Otros (Beraldo et al., 1996) destacan la mineralización de la fibra por inmersión previa en soluciones a base de silicato de sodio y sulfato de aluminio.

Ya en la presente línea de investigación, el problema de la durabilidad de las fibras fue resuelto con el empleo de matrices de baja alcalinidad (agua en los poros de cuerpo de prueba con $\mathrm{pH}$ inferior a 9), tales como yeso y cementos de escoria granulada básica de alto horno. Por los estudios disponibles, se confirma la viabilidad de componentes constructivos resultantes, con durabilidad semejante a la de otros sistemas constructivos compatibles (Agopyan y John, 1992).

\section{Trabajo experimental}

\section{Investigación de disponibilidad y selección de residuos}

A continuación una síntesis de las regiones visitadas, en el período de enero a agosto de 1997, con el objeto de analizar el proceso de cultivo, extracción, beneficio e industrialización de 
fibras vegetales, teniendo en cuenta la respectiva identificación y cuantificación de todos los residuos generados. Fueron, en total, 23 empresas visitadas y 15 entidades de extensión y/o pesquisa, así localizadas:

- Norte de Paraná: regiones productoras y procesadoras de rami (Boemmiria nivea).

- Valle de Ribeira SP: producción de banana (Musa cavendishii).

- Aracruz ES: fábrica de pulpa de celulosa de eucalipto (Eucalyptus grandis - clones), para producción de papel.

- Bahía y Paraiba: producción y procesamiento de la fibra de sisal (Agave sisalana).

- Pernambuco, Sergipe, Ceará e interior de São Paulo: producción de coco (Cocos nucifera) y procesamiento de la fibra extraída del fruto.

- Valença BA: extracción y procesamiento de la fibra de piaçava (Attalea funifera).

- Pará: regiones productoras y procesadoras de malva (Urena lobata).

Se procedió a la caracterización y cuantificación de los residuos a partir de muestras recolectadas, además de documentación fotográfica. A partir de esas informaciones obtenidas en los viajes técnicos, se procedió a la clasificación de los residuos, con los siguientes criterios de selección:

- Identificación general de la producción agroindustrial generadora de residuos: caracterización de los productos principales, región productora, cantidades producidas y operaciones envueltas.

- Identificación de los residuos: relación con productos principales, procesos y operaciones.

- Cantidad disponible de residuos: otras opciones de uso, con las demandas respectivas.

- Distribución de los residuos generados: adaptación a soluciones regionalizadas y costos de transporte.

- Valor de mercado del residuo.

- Caracterización de las materias primas y de los compósitos producidos.

La tabla $\mathrm{N}^{\circ} 1$ contiene las principales propiedades físicas y mecánicas, referentes a las fibras vegetales de mayor interés. 
Siguen también informaciones referentes a la fibra de polipropileno, a manera de comparación. Es importante resaltar que las propiedades de las fibras presentan coeficientes de variación altos, frecuentemente mayores a 40\%. Aunque presenten elevada resistencia a la tracción, el módulo de elasticidad de las fibras vegetales es menor que el de las matrices cementicias (de 20 a $30 \mathrm{GPa})$.

Tabla $\mathbf{N}^{\circ} 1$

Características físicas y mecánicas de algunas fibras vegetales

\begin{tabular}{|c|c|c|c|c|}
\hline Tipo de fibra & $\begin{array}{l}\text { Patrón sin } \\
\text { fibra }\end{array}$ & $\begin{array}{l}\text { Sisal-desecho } \\
\text { de campo }\end{array}$ & $\begin{array}{c}\text { Pulpa de celulosa } \\
\text { de eucalipto }\end{array}$ & $\begin{array}{c}\text { Coco-polvo } \\
\text { residual }\end{array}$ \\
\hline $\begin{array}{l}\text { Masa específica en el } \\
\text { estado recién-mixturado } \\
\left(\mathrm{kg} / \mathrm{m}^{3}\right)\end{array}$ & 2094,4 & 2051,9 & 2067,6 & 2020,6 \\
\hline Índice de consistencia (mm) & 322,0 & 192,4 & 164,1 & 235,4 \\
\hline $\begin{array}{l}\text { Absorción de agua (\% en } \\
\text { masa) }\end{array}$ & 14,73 & 10,43 & 15,12 & 13,11 \\
\hline $\begin{array}{l}\text { Resist. a la tracción en la } \\
\text { flexión a los } 42 \text { días (mpa) }\end{array}$ & 3,41 & 4,32 & 4,46 & 3,85 \\
\hline $\begin{array}{l}\text { Ens. de fractura en la } \\
\text { flexión a los } 42 \text { días (n.mm) }\end{array}$ & 173 & 515 & 567 & 602 \\
\hline $\begin{array}{l}\text { Resist. a la compresión a } \\
\text { los } 28 \text { días (mna) }\end{array}$ & 20,38 & 22,32 & 15,12 & 24,56 \\
\hline
\end{tabular}

Análisis de los compósitos fibrosos

En conjunto con otros proyectos de investigación en marcha, como a partir de informaciones de experimentos reportados en la bibliografía consultada (Agopyan, 1991 y John, 1995), se llegó a la definición del aglomerante cementicio y del compósito a ser probado en esta fase experimental. Fueron amoldados compósitos, con matriz de argamasa, según los siguientes procedimientos: 
- Relación aglomerante: arena gruesa: 1 : 1,5.

- Relación agua / aglomerante: 0,5.

- Tenor en volumen de fibra: $2 \%$.

- Tipos de fibra: conforme a la preselección llevada a cabo.

- Aglomerante: cemento de escoria básica granulada de alto horno (procedencia: CST, Blaine: $500 \mathrm{~m}^{2} / \mathrm{kg}$ ), activada por gipsita e hidróxido de calcio. Sigue, en la tabla $\mathrm{N}^{\mathrm{o}}$ 2, la composición de óxidos de la escoria, mediante el empleo del difractómetro de rayos $\mathrm{X}$.

- Mezclado y moldeado manual del compósito. Cura por inmersión en agua, durante siete días, y al aire (ambiente de laboratorio) hasta el día de los ensayos.

- Ensayos en el estado recién-mixturado: masa específica (NBR 9833) e índice de consistencia (NBR 7215).

- Ensayos en el estado endurecido: compresión axial (CP 50x100mm cilíndrico-NBR 5739), tracción por compresión diametral (CP 50x100mm cilíndrico-NBR 7222), tracción en la flexión en un dispositivo con cuatro apoyos (CP 300x150x15mm prismático-Rilem 49TFR) y absorción de agua por inmersión (NBR 9778).

Tabla $\mathbf{N}^{\circ} 2$

Composición química de la escoria de alto horno

$\begin{array}{lllllllllll}\text { Com- } & \mathrm{SiO}_{2} & \mathrm{Al}_{2} \mathrm{O}_{3} & \mathrm{Fe}_{2} \mathrm{O}_{3} & \mathrm{MnO} & \mathrm{MgO} & \mathrm{CaO} & \mathrm{Na}_{2} \mathrm{O} & \mathrm{K}_{2} \mathrm{O} & \mathrm{TiO}_{2} & \mathrm{P}_{2} \mathrm{O}_{5}\end{array}$ puesto

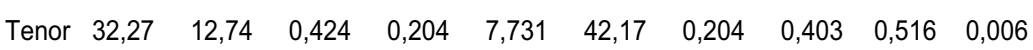

(\%)

\section{Resultados}

Residuos preseleccionados

Basándonos en la investigación de campo fueron preseleccionados algunos residuos, en condiciones de disponibilidad inmediata, para uso en la construcción civil:

- Desecho de campo del sisal. Gran disponibilidad y bajo interés comercial, además de ser alternativa de aumen- 
to de ganancias para los productores agrícolas. Existe la necesidad de que la fibra pase por limpieza (malla cilíndrica-gaiola).

- Desecho de máquina de la producción de empaques trenzados. Fibras exentas de polvo residual y producción concentrada en pequeño número de empresas, lo que facilita su utilización. Pero el tratamiento utilizado es a base de aceite mineral, pudiendo afectar las propiedades mecánicas de la fibra y la adherencia fibra-matriz.

- Fibrillas extraídas del polvo residual del coco. Valor de mercado reducido, con gran posibilidad de producción y aprovechamiento actual casi nulo. Entretanto necesita de separación del polvo (cerca de $50 \%$ en masa) y secado.

- Desecho de celulosa de eucalipto. Valor de mercado casi nulo y gran disponibilidad. Desventaja: la pequeña longitud de las fibras, del orden de 1 a $2 \mathrm{~mm}$.

Otro residuo también está siendo considerado como parámetro, por su potencialidad de uso futuro:

- Fibra del tallo de la bananera. Gran disponibilidad, desde que es extraída por procesos elementales. Posible fuente alternativa de renta, en región de poco desarrollo económico de São Paulo, y al mismo tiempo próxima a grandes centros urbanos.

La tabla $\mathrm{N}^{\mathrm{o}} 3$, elaborada a partir de Savastano Jr. et al. (1997), contiene las principales informaciones de interés al respecto de los residuos arriba mencionados. Las muestras preseleccionadas fueron analizadas por microscopía electrónica de barrido, en el CME-Esalq-USP, con algunos resultados disponibles en las figuras $\mathrm{N}^{\mathrm{o}} 1-4$. 
Tabla $\mathbf{N}^{\circ} 3$

Algunos residuos originados en el procesamiento de fibras vegetales

\begin{tabular}{|c|c|c|c|c|}
\hline Tipo de fibra & $\begin{array}{c}\text { Patrón sin } \\
\text { fibra }\end{array}$ & $\begin{array}{c}\text { Sisal-desecho } \\
\text { de campo }\end{array}$ & $\begin{array}{c}\begin{array}{c}\text { Pulpa de celulosa } \\
\text { de eucalipto }\end{array} \\
\end{array}$ & $\begin{array}{c}\text { Coco-polvo } \\
\text { residual }\end{array}$ \\
\hline $\begin{array}{l}\text { Masa específica en el } \\
\text { estado recién-mixturado } \\
\left(\mathrm{kg} / \mathrm{m}^{3}\right)\end{array}$ & 2094,4 & 2051,9 & 2067,6 & 2020,6 \\
\hline Índice de consistencia (mm) & 322,0 & 192,4 & 164,1 & 235,4 \\
\hline $\begin{array}{l}\text { Absorción de agua (\% en } \\
\text { masa) }\end{array}$ & 14,73 & 10,43 & 15,12 & 13,11 \\
\hline $\begin{array}{l}\text { Resist. a la tracción en la } \\
\text { flexión a los } 42 \text { días (mpa) }\end{array}$ & 3,41 & 4,32 & 4,46 & 3,85 \\
\hline $\begin{array}{l}\text { Ens. de fractura en la } \\
\text { flexión a los } 42 \text { días (n.mm) }\end{array}$ & 173 & 515 & 567 & 602 \\
\hline $\begin{array}{l}\text { Resist. a la compresión a } \\
\text { los } 28 \text { días (mpa) }\end{array}$ & 20,38 & 22,32 & 15,12 & 24,56 \\
\hline 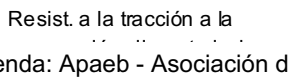 & Pequeño & cultores del M & ipio de $\hat{\text { Valente } B A}$ & $\cdots$ \\
\hline
\end{tabular}

Aracruz Celulose S.A. - Aracruz ES.

La microscopía del desecho verde del sisal (figura $\mathrm{N}^{0} 1$ ) muestra la fibra recubierta por mucilagem (que puede actuar como retardador de la unión de aglomerantes cementicios) y todavía con fibrillas y estrías en el sentido longitudinal. El desecho de empaques trenzados, visto en la figura $\mathrm{N}^{\circ} 2$ ya presenta una gran alteración superficial de la fibra, en vista de los procesos mecánicos y de tratamiento con aceite mineral. La fibra de coco (véase figura $\mathrm{N}^{\circ} 3$ ) posee forma cilíndrica con protuberancias superficiales que ayudan en la fijación de la fibra en las matrices reforzadas. Por último, el desecho de celulosa (véase figura $\mathrm{N}^{\mathrm{o}}$ 4) presenta morfología diferenciada, con fibras semejantes a cintas fibriladas y bastante alteradas por los procesos químico y mecánico, asociados a la producción de la pulpa. 
Figura $\mathrm{N}^{\circ} 1$

Desecho verde de sisal del campo

\begin{tabular}{lccr}
\hline \multicolumn{1}{c}{ Tipo de fibra } & $\begin{array}{c}\text { Patrón sin } \\
\text { fibra }\end{array}$ & $\begin{array}{c}\text { Sisal-desecho } \\
\text { de cam po }\end{array}$ & $\begin{array}{r}\text { Pulpa de } \\
\text { de eu }\end{array}$ \\
\hline $\begin{array}{l}\text { Masa específica en el } \\
\text { estado recién-mixturado } \\
\left(\mathrm{kg} / \mathrm{m}^{3}\right)\end{array}$ & 2094,4 & 2051,9 & 20 ' \\
$\begin{array}{l}\text { Índice de consistencia }(\mathrm{mm}) \\
\begin{array}{l}\text { Absorción de agua }(\% \text { en } \\
\text { masa) }\end{array}\end{array}$ & 322,0 & 192,4 & 16 \\
$\begin{array}{l}\text { Resist. a la tracción en la } \\
\text { flexión a los } 42 \text { días }(\mathrm{mpa})\end{array}$ & 14,73 & & 10,43 \\
\end{tabular}

Figura $\mathrm{N}^{\circ} 2$

Desecho de empaque trenzado (hilo agrícola)

\begin{tabular}{lccc}
\hline \multicolumn{1}{c}{ Tipo de fibra } & $\begin{array}{c}\text { Patrón sin } \\
\text { fibra }\end{array}$ & $\begin{array}{c}\text { Sisal-desecho } \\
\text { de cam po }\end{array}$ & $\begin{array}{r}\text { Pulpa de celulo } \\
\text { de eucalipto }\end{array}$ \\
\hline $\begin{array}{l}\text { Masa específica en el } \\
\text { estado recién-mixturado } \\
\left(\mathrm{kg} / \mathrm{m}^{3}\right)\end{array}$ & 2094,4 & 2051,9 & 2067,6 \\
& & & 164,1 \\
Índice de consistencia $(\mathrm{mm})$ & 322,0 & 192,4 & 15,12 \\
$\begin{array}{l}\text { Absorción de agua (\% en } \\
\text { masa) }\end{array}$ & 14,73 & 10,43 & \\
$\begin{array}{l}\text { Resist. a la tracción en la } \\
\text { flexión a los 42 días (mpa) }\end{array}$ & 3,41 & 4,32 & 4,46
\end{tabular}


Figura $N^{\circ} 3$

Fibrillas de polvo residual de coco

\begin{tabular}{lccc}
\hline \multicolumn{1}{c}{ Tipo de fibra } & $\begin{array}{c}\text { Patrón } \mathbf{s i n} \\
\text { fibra }\end{array}$ & $\begin{array}{c}\text { Sisal-desecho } \\
\text { de cam po }\end{array}$ & $\begin{array}{c}\text { Pulpa de celulosa } \\
\text { de eucalipto }\end{array}$ \\
\hline $\begin{array}{l}\text { Masa específica en el } \\
\text { estado recién-mixturado } \\
\left(\mathrm{kg} / \mathrm{m}^{3}\right)\end{array}$ & 2094,4 & 2051,9 & 2067,6 \\
& & & 164,1 \\
Índice de consistencia (mm) & 322,0 & 192,4 & 15,12 \\
$\begin{array}{l}\text { Absorción de agua (\% en } \\
\text { masa) }\end{array}$ & 14,73 & 10,43 & \\
$\begin{array}{l}\text { Resist. a la tracción en la } \\
\text { flexión a los } 42 \text { días (mpa) }\end{array}$ & 3,41 & 4,32 & 4,46 \\
. . . . & & &
\end{tabular}

Figura $N^{\circ} 4$

Desecho de celulosa de eucalipto

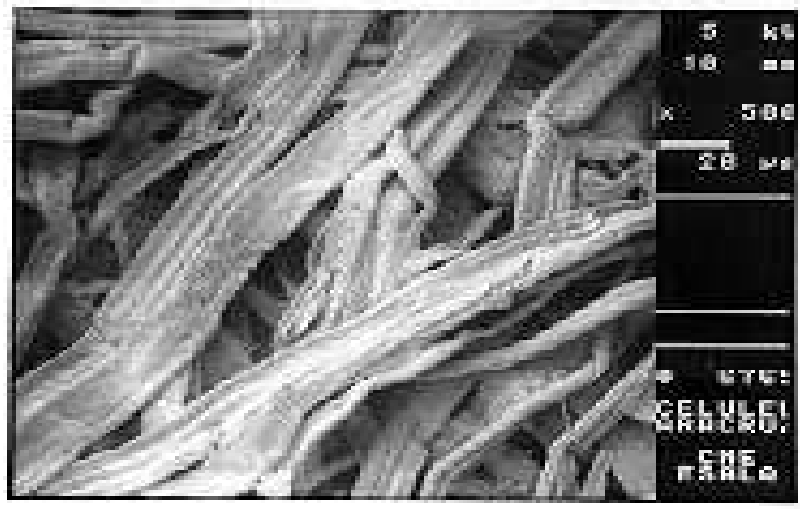


Resultados de los ensayos físicos y mecánicos

A continuación, en la tabla $\mathrm{N}^{0} 4$, informaciones referentes a ensayos físicos (estado recién-mixturado y a los 28 días de edad) y mecánicos (28 y 42 días de edad). La resistencia a la tracción en la flexión del compósito reforzado con fibras de celulosa residual de eucalipto fue $30 \%$ superior a la del patrón. La energía de fractura en la flexión corresponde al total de energía absorbida, hasta la reducción de la carga equivalente a 70\% de la carga máxima: ese resultado muestra la gran diferencia de los compósitos fibrosos, en relación con la matriz sin fibras, ya en el estado posmixturado.

Tabla $N^{\circ} 4$

Propiedades de los compósitos a base de cemento de escoria de alto horno

\begin{tabular}{|c|c|c|c|c|}
\hline Tipo de fibra & $\begin{array}{c}\text { Patrón sin } \\
\text { fibra }\end{array}$ & $\begin{array}{c}\text { Sisal-desecho } \\
\text { de campo }\end{array}$ & $\begin{array}{l}\text { Pulpa de celulosa } \\
\text { de eucalipto }\end{array}$ & $\begin{array}{c}\text { Coco-polvo } \\
\text { residual }\end{array}$ \\
\hline $\begin{array}{l}\text { Masa específica en el } \\
\text { estado recién-mixturado } \\
\left(\mathrm{kg} / \mathrm{m}^{3}\right)\end{array}$ & 2094,4 & 2051,9 & 2067,6 & 2020,6 \\
\hline Índice de consistencia $(\mathrm{mm})$ & 322,0 & 192,4 & 164,1 & 235,4 \\
\hline $\begin{array}{l}\text { Absorción de agua (\% en } \\
\text { masa) }\end{array}$ & 14,73 & 10,43 & 15,12 & 13,11 \\
\hline $\begin{array}{l}\text { Resist. a la tracción en la } \\
\text { flexión a los } 42 \text { días (mpa) }\end{array}$ & 3,41 & 4,32 & 4,46 & 3,85 \\
\hline $\begin{array}{l}\text { Ens. de fractura en la } \\
\text { flexión a los } 42 \text { días (n.mm) }\end{array}$ & 173 & 515 & 567 & 602 \\
\hline $\begin{array}{l}\text { Resist. a la compresión a } \\
\text { los } 28 \text { días (mpa) }\end{array}$ & 20,38 & 22,32 & 15,12 & 24,56 \\
\hline $\begin{array}{l}\text { Resist. a la tracción a la } \\
\text { compresión diametral a los } \\
28 \text { días (mpa) }\end{array}$ & 2,72 & 3,39 & 2,92 & 2,83 \\
\hline
\end{tabular}


Los criterios utilizados resaltaron la viabilidad del uso de los residuos preseleccionados de fibras vegetales, principalmente basados en la gran disponibilidad y bajo valor de mercado (alta cantidad de fibras plásticas de bajo módulo de elasticidad disponibles comercialmente). Con relación a los resultados obtenidos hasta el momento, para los ensayos mecánicos de tracción y compresión, los compósitos fibrosos a base de cemento de escoria de alto horno presentaron desempeño, en la mayoría de los casos, superior al patrón de referencia. La capacidad de refuerzo de las fibras vegetales se mostró más significativa en los ensayos de flexión, con resistencia a la tracción y energía de fractura muy superiores al patrón, aun sin el empleo de técnicas más costosas de moldeo, espesamiento y cura de los compósitos.

\section{Bibliografía}

Agopyan, V.

"Materiais reforçados com fibras para a construção civil nos países em desenvolvimento: uso de fibras vegetais". Tese (Livre-Docência)- Escola Politécnica. São Paulo: Universidade de São Paulo, 1991, 204 pp.

Agopyan, V.; V.M. John

"Durability Evaluation of Vegetable Fibre Reinforced Materials". Building Research and Information. Vol. 20, No 4, 1992, pp. 233-235.

Agopyan, V.; H. Savastano Jr.

"Uso de materiais alternativos à base de fibras vegetais na construção civil: experiência brasileira". Seminario Iberoamericano 1997 de Materiales Fibrorreforzados 1 y Reunion del Proyecto PIP VIII.5 Cyted, 3. Cali, 1997. Memorias. Cali, Cyted/Universidad del Valle, 1997, pp. 23-40 (artículo 3).

Beraldo, A.L. et al.

"Viabilidade de fabricação de compósitos resíduos de madeiras e cimento Portland (CBC)". Reciclagem e reutilização de resíduos como materiais de construção civil. São Paulo: Anais, 1996. São Paulo, EP-USP/Antac, 1997, pp. 77-82. 